die Bedeutung von Politik und Wirtschaft aus geteilten Erfahrungen zusammenzutragen, und der Austausch und das gemeinsame Treffen von Entscheidungen sind vielfältiger geworden.

Diese soziokulturellen Transformationen eröffnen den textbasierten und audiovisuellen Medien sowie den Netzwerken Möglichkeiten zur Aufdeckung von Korruption und anderen politischen Verstrickungen, was eine nie zuvor dagewesene Auswirkung auf die Masse hat. Das Vergleichen von Meldungen und ihren verschiedenen Versionen erweitert den Sinn und macht ihn pluralistischer und polemischer. Die vielfältigen Medien von heute befähigen die Bürger zum Herauslesen von Indizien und Verbindungen zwischen Texten, die ehemals getrennt in den Rubriken für Politik, Wirtschaft, Kultur und Unterhaltung präsentiert wurden.

\title{
Apps vs. Institutionen
}

Der technologiebezogene Wortschatz hat uns so viele neue Namen beschert, dass es schwer ist, die Relevanz jedes einzelnen nachzuvollziehen. Sind wir digitale Junkies, denen nahegelegt wird, immer verbunden zu sein? Allmächtige Wesen, dank der unaufhaltbaren Ausweitung unserer Kontakte? Verlinkt, aber auch blockierbar? Hyperinformierte Menschen, denen es schwerfällt, zwischen der Wahrheit und dem Unwahren, zwischen echten Personen und Bots zu unterscheiden?

Apps geben vor, uns das Leben zu erleichtern - sie wählen die kürzeste Reiseroute mit wenig Verkehr, schlagen uns Freunde als Begleiter bei der Arbeit und in der Freizeit vor, und vieles mehr. Trotzdem helfen sie uns nicht dabei weiter, zu verstehen, warum wir das alles tun. Die Apps sind wie unsere Begleiter: Ist das ihr Sinn? Was ist mit unseren Mitmenschen? Es wird immer verzichtbarer, sich mit Freunden zu treffen, wenn wir uns Nachrichten schicken können, auch wenn dafür der Wunsch nach ihrer ständigen Verfügbarkeit auf WhatsApp und die Bedingung, dass sie uns auch antworten, erfüllt sein muss. Wir können von uns Selfies schießen, ohne dass uns jemand hilft, uns im Bild einfängt und uns zu einer »spontanen« Mimik für das Foto anregt. Dabei entfallen auch die Bewunderung oder die Lacher, wenn wir das Bildresultat des einmaligen Klicks gemeinsam betrachten. Ein frischer, unmittelbarer Eindruck kann heute auch durch Fotoretusche herbeigezaubert werden. Aber es ist doch so, dass viele Selfies gemacht werden, um sie an einen bestimmten Empfänger zu bringen. 
Die anderen sind da und schlendern digital durch unsere Tätigkeiten, die wir teilen. In gewisser Weise - sagten früher die Philosophen und Psychoanalytiker - führen wir sie mit uns mit. Und das nicht im losen Sinne, wie etwa den Vater, die Mutter, Geschwister und den Partner oder gar den Arbeitskollegen, sondern als Institutionen - die Familie oder die Fabrik. Bisher sind noch keine Studien bekannt, die untersuchen, wie diese Institutionen mit den Apps konkurrieren. Ein Komiker greift die Idee jedoch auf einmal auf. Andrés Rábago, auch bekannt als El Roto, hat einen Teenager gespielt, der gerade dabei ist, mit seinem Finger den Handybildschirm zu berühren und dabei sagt: »Eltern sind eine Handy-App«.

Zusammen mit den schriftlichen und visuellen Botschaften, die auf Distanz in Echtzeit ausgetauscht werden und durch die ein Gefühl der KoPräsenz entsteht, führt die Vorherrschaft der Smartphones und der E-Mails $\mathrm{zu}$ Veränderungen in der Interaktion und der Verfügbarkeit für unsere Mitmenschen. Nicht nur persönliche Treffen werden ersetzt, wenn Vereinbarungen per E-Mail und WhatsApp-Nachricht getroffen werden. Auch die Stimme des anderen verschwindet auf diese Weise zunehmend. Außerdem kann es vorkommen, dass wir stunden- oder tagelang auf eine Antwort warten, sodass die Unmittelbarkeit nicht mehr gegeben ist und der Gesprächspartner die Möglichkeit hat, seine Antwort genauer auszufeilen und so die gewöhnlichen Effekte, die sich aus der Stimmlage und ganz besonders aus der Konfrontation von Angesicht zu Angesicht ergeben, zu manipulieren. Mehr Freiheit für jedes Subjekt, mehr Distanz, eine geringere Verbindlichkeit? Verliert sich die Intensität des Schweigens und der gelegentlichen Leere im Gespräch, oder ergeben sich andere Akzente und Anregungen durch das Schreiben und Lesen? Die Annäherung, die durch diese neuen Vermittler generiert wird, ist gleichzeitig eine Entfernung - die Nähe kann zur Distanz führen.

Die Beziehung zu unseren Mitmenschen wurde einst durch die Institutionen geschlichtet oder in einem Rahmen gehalten. Sie waren, offensichtlich oder nicht, der Beweis dafür, dass wir begleitet wurden. Wie rekonfigurieren sich persönliche Zustände wie Zuneigung, Gewissheit und Not, wenn wir auf Apps zurückgreifen, bezahlen und bezahlt werden, und unsere medizinischen Testergebnisse über Bildschirme erfahren?

Wenn Mobiltelefone zum Sprechen dienen, sei es durch aufgeschobene Kommunikation wie in den Chats, und wenn Verabredungen schnell vereinbart werden oder wir uns zumindest in Konversationen auf Distanz miteinander »treffen« und wissen, dass die anderen da sind, was sie denken und 
was sie bewegt, dann fühlen wir alltäglich, dass die Apps die veralteten Institutionen (Parteien, Kirchen, Gewerkschaften) ersetzen können.

Dieses Vergnügen existiert parallel zu zahlreichen Unannehmlichkeiten. Zum einen muss man zwischen den alten Institutionen und den jüngsten virtuellen Aktivitäten interagieren. Ein weiteres Hindernis ergibt sich aus dem Wissen, dass wir ständig abgefangen und unsere Daten, Fotos oder Reaktionen an geheimen Orten verwertet werden. Insofern scheint es schwieriger geworden zu sein, dieses Eindringen, den digitalen Terrorismus und die Entführung von Informationen, Geld und Sicherheit zu kontrollieren, als Banken oder Flughäfen, in denen Handys nicht benutzt werden sollen.

Selbstbestimmung und Enteignung gehen miteinander einher. Die Fähigkeit zum Umgang mit diesem Widerspruch, durch den wir etwas erhalten und uns im Gegenzug gleichzeitig etwas weggenommen wird, ist die Aufgabe der Bürger: nicht einfach nur Süchtiger oder Follower zu sein. Immer mehr Autoren, darunter Ronald J. Deibert, Evgeny Morozov und Eric Sadin, argumentieren jedoch, dass das, was von den alten Formen der Staatsbürgerschaft übriggeblieben ist, von geringem Nutzen sei. Demnach befinden wir uns immer noch in einem Prozess des Herausfindens über die Frage, ob diese Form des Lebens einen neuen Sinn annehmen kann, der inmitten der Raster der Algorithmen weniger abstrakt und dafür pluralistischer wäre.

Die Rede ist vom elektronischen, kognitiven Kapitalismus, sowie vom Tech-Kapitalismus. Dazu muss betont werden, dass zusätzlich zu den Veränderungen in den Interaktionen zwischen Parteien, Videopolitik, digitalen Technologien, Unternehmen und Nutzern der derzeitige unklare Zustand der Staatsbürgerschaft auf die Mutation der Biopolitik zurückgeht. Neben ihrer soziokulturellen Bedeutung charakterisieren sich der gegenwärtige Kapitalismus und die Strategien zur Transformation oder zum Widerstand durch etwas, was in der Kultur als Symbolsystem eine zentrale Bedeutung hat: die Zuneigung. George Yúdice beschreibt den Kapitalismus der Gegenwart als »Ökonomie der Information, der Erfahrung und der Zuneigung« (Yúdice 2016). Dass wir die Rolle von Suchtverhalten, Kontakten, sozialen Netzwerken und dem Akt des Verlinkens und Blockierens diskutieren, liegt daran, dass die Subjektivität und der Körper in unseren täglichen Verhaltensweisen impliziert sind. Diese weniger greifbaren Verhaltensweisen haben eine Schlüsselbedeutung für den Aufbau des Handelns bzw. der Staatsbürgerschaft erhalten, sowohl in Bezug auf offen politische, als auch öffentliche Handlungen empörter Bürger. Sie zeigen sich etwa in Austauschbewegungen und neuen Formen der Zugehörigkeit, die uns einer Bewegung näherbringt 
und uns von den fest verankerten oder korrupten Organisationen wegbringt, die oft in Form von Parteien existieren.

Es gibt einen Grund dafür, dass wir von der »Abkehr« von der Politik sprechen. Laut Yúdice brauchen wir eine »kritische Perspektive auf die auf Zuneigung basierenden Technologien«, anstatt bloß die Analyse und Sammlung der Daten zu betrachten. Dann könnten wir verstehen, wie die Plattformen sie nutzen, ohne dass bei uns ein Gefühl der Unterwerfung oder Unterdrückung entsteht. "Die große Mehrheit liest nicht einmal die Nutzungsbedingungen, akzeptiert die AGB für den schnellen Zugang, den eigenen Ausdruck und die Vernetzung. Es ist genau dieser Imperativ des Sich-Ausdrückens, der in sozialen Netzwerken vorherrscht, und der dem auf Erfahrung und Zuneigung gründenden Kapitalismus zugutekommt « (Idem).

Zuneigungen sind mit Wünschen verbunden (und lösen deshalb Reaktionen gegen die kapitalistische Unterdrückung aus, die uns daran hindert, unsere Wünsche zu verwirklichen). Über Zuneigung entstehen auch Überzeugungen, etwa gegenüber Bewegungen, Parteien oder Anführern, die versprechen, den Weg für unsere Ziele zu ebnen. Die Psychoanalyse ist als Disziplin, die Instrumente zur Interpretation dieser drei Prozesse liefert, hilfreich, wenn sie sich für soziale Themen öffnet und dadurch kollektive Projekte und deren transformatorisches Potenzial, sowie Anteile der Frustration und der Illusion in ihnen nachvollzieht. Sie kann auch kritisch beleuchten, wie unsere Beziehung zu den Algorithmen funktioniert. Sie sind zum einen Kollektoren unserer Wünsche und Überzeugungen im Rahmen bewusster Manifestationen. Zum anderen wirken sie ähnlich wie politische Anführer: sie wirken wie Bildschirme, auf die wir eine imaginäre Bedeutung projizieren. Die interpretative und kritische Kapazität der Psychoanalyse ergibt sich aus der Wissensproduktion über die verborgenen Mechanismen, die uns dazu führen, kollektive Hoffnungen zu entwickeln. Ebenso wie manche Autoren die wünschenswerte Dimension der kollektiven Überzeugungen mit dem verknüpfen, was die soziopolitische Theorie an utopischen Projekten, Anführern und algorithmischem Wissen kritisiert, ist es möglich, die psychosoziale Natur der Emanzipationsund Unterwerfungsprozesse zu verstehen. Sie bieten auch Instrumente zum Verständnis darüber, wie die Suche nach Sinn und Vergnügen mit den intensiven Aktionen und Gefühlen verstrickt sind, die bei Revolten zu Stande kommen (Zigouris 2005).

Anhand dieser Schlüsselpunkte ergibt sich eine Lesemöglichkeit unbeugsamer lateinamerikanische Bewegungen, die sich der neoliberalen Politik widersetzen. In ihren Aktionen und Slogans zeigen sich nicht bloß utopische 
Gedanken. Beispiele sind die Landlosen in Brasilien, der mexikanische Zapatismus, die indigenen Rebellionen in Bolivien und Ecuador, die Nachkommen der Verschwundenen oder besetzte Fabriken in Argentinien: »eine andere Welt ist möglich«, »eine Welt, in die viele Welten passen«, »besetzen, widerstehen, produzieren«. Ihre Stärke und Beständigkeit hängen von der Fähigkeit ab, sich über die Organisation von Parteien und Gewerkschaften hinaus neu zu erfinden. Dafür braucht es andere soziopolitische Konstruktionen über eine direkte Demokratie, Horizontalität und Selbstverwaltung. Angesichts der ernüchternden Realität scheint

die Willenskraft dieser sozialen und politischen Bewegungen [...] heutzutage mit einer Vielfalt an Handlungsformen zu verschmelzen, die kollektiv agieren, um hier und jetzt Existenzbedingungen zu ändern. Es handelt sich um territoriale Bewegungen. Sie nehmen vernachlässigte Räume ein und nehmen sie in Produktion; Felder zum Besäen, Häuser zum Bewohnen, Fabriken zur Herstellung. Sie befinden sich nicht nur Widerstand, sondern erfinden sich ihre Welt selbst. Es sind zwar kleine Welten, aber eigene. (Fernández 2010)

Nach dieser intensiven Periode der Apps beobachten wir damit einen Wunsch nach Institutionen. Kollektive Aktionen haben die Grenzen des Möglichen ausgeweitet. Erwähnt seien die Errungenschaften von Menschenrechtsbewegungen und die Schließung von 220 Fabriken nach der argentinischen Krise im Jahr 2001, die von 1500 Arbeitern in einer autonomen Aktion wiedergeöffnet wurden und schließlich in Selbstverwaltung wettbewerbsfähig wurden. Frustrationen werden jedoch auch deutlich. Um diese scheinbar widersprüchlichen Ergebnisse zu verstehen bedarf es Studien, die sich mit der $\mathrm{Zu}$ neigung, Wünschen und Überzeugungen beschäftigen, die von einem erneuerten sozioökonomischen und politischen Denken begleitet werden.

Hier kehre ich zum Wert der Indexmethode zurück, die auch von denjenigen benutzt wird, die nicht wissen, wer Clifford Geertz oder Carlo Ginzburg sind, aber deren Taktiken die Fähigkeit haben, im Wissen um das Ganze (also im neoliberalen und elektronischen Kapitalismus) zwischen den Zeilen zu lesen und Lücken zu erkennen, sodass kritische Stimmen diese Mängel ausnutzen und sie sich durch Störaktionen zu Nutze machen können. Wie weit können sie als alternativer Weg des Wissens kommen? Meine hervorgebrachte Synthese, deren Ausarbeitung an dieser Stelle den Rahmen sprengen würde, lautet: Das Indizienparadigma ist nützlicher, wenn es um das Entdecken unbekannter Spuren geht. Aber in Kontexten der Rechtfertigung und Anwen- 
160 Demokratie im digitalen Kapitalismus

dung sind andere Wissensformen vonnöten, die eine ganzheitliche Perspektive einnehmen und Handlungsoptionen eröffnen, die nicht auf den Status der Ausnahme beschränkt bleiben. 